\title{
Efectos del ejercicio físico sobre la depresión y la ansiedad
}

\author{
Cristhian Felipe Delgado Salazar ${ }^{a}$ \\ Erika Tatiana Mateus Aguilera ${ }^{a}$ \\ Laura Alejandra Rincón Bolivara \\ Wilder Andres Villamil Parra ${ }^{\text {ab } 1}$ \\ https://orcid.org/0000-0002-1717-1020 \\ a Escuela Colombiana de Rehabilitación \\ b Universidad Nacional de Colombia
}

Fecha de recepción: 12 de abril de 2019

Fecha de aprobación: 17 de junio de 2019

\begin{abstract}
Resumen
Los trastornos de ansiedad y depresión son el mayor problema de salud mental a nivel mundial. Puede presentarlos cualquier persona, provocando alteraciones biológicas y psicológicas que afectan las actividades de la vida diaria. Método: Revisión sistemática basada en la búsqueda de ECAS que vincularon el ejercicio físico como estrategia terapéutica para estos trastornos. Se realizó una búsqueda en las bases de datos: Redalyc, PubMed, EBSCO host, Elsevier, PeDro, DialNet, Scielo y ATGC (Pubmed) con punto de corte Marzo 2018. Se analizaron de 136 artículos y se evaluaron con la lista de chequeo CONSORT creando 3 matrices de análisis cuantitativo, en donde se resalta la incidencia de trastornos de depresión y ansiedad en los grupos etarios de juventud, adultez y adulto mayor, identificando los factores por estrés post traumático, origen ocupacional, drogodependencia, trastornos mentales, enfermedades diagnosticadas, conductas según la edad y ginecobstetricas. Resultados: El ejercicio físico realizado de manera estructurada disminuye significativamente los síntomas depresivos $(\mathrm{p} \leq 0.001)$ evaluado con diversas escalas entre las que se destacan las pruebas Inventario de Beck, Escala de Depresión Geriátrica (GDS), DASS-21 y BDI. La reducción en el nivel de ansiedad, evaluado con la escala HADS ( $\mathrm{p}=10.0007$ ) en todos los subgrupos, resaltando la prescripción individualizada Discusión: Se confirman los efectos positivos del ejercicio físico prescrito, en los síntomas de depresión y ansiedad de diversas poblaciones, siendo efectiva y de bajo costo, lo que abre la necesidad de priorizarlo y aplicar sus principios a actividades complementarias como el taichi y/o yoga para mejorar su efectividad.
\end{abstract}

Palabras clave: Ansiedad; depresión; ejercicio; fisioterapia; salud mental; trastorno de la conducta.

\footnotetext{
${ }^{1}$ Correo de contacto: wavillamilp@unal.edu.co DOI: 10.30788/RevColReh.v18.n2.2019.389
}

Esta obra se encuentra bajo licencia internacional CC BY 4.0 Institución editora: Escuela Colombiana de Rehabilitación 


\title{
Effects of physical exercise on depression and anxiety
}

\begin{abstract}
Anxiety disorders and depression are the biggest problem of mental health in the world. It can be present by anyone, causing biological and psychological alterations that affect the activities of daily life. Method: Systematic review based on the search for ECAS that linked physical exercise as a therapeutic strategy for these disorders. A search was performed on the databases: Redalyc, PubMed, EBSCO host, Elsevier, PeDro, DialNet, Scielo and ATGC (Pubmed) with cut-off point March 2018. 136 articles were analyzed and evaluated with the CONSORT checklist creating 3 formats of quantitative analysis, highlighting the incidence of depression and anxiety disorders in the age groups of youth, adulthood and older adulthood, identifying post-traumatic stress factors, occupational origin, drug dependence, mental disorders, diagnosed illnesses, age-based behaviors and gynecology.

Results: Physical exercise performed in a structured way significantly decreases depressive symptoms $(\mathrm{p} \leq 0.001)$ evaluated on a number of scales including Beck Inventory, Geriatric Depression Scale (GDS), DASS-21 and BDI tests. The reduction in the level of anxiety, assessed with the HADS scale $(\mathrm{p}=10.0007)$ in all subgroups, highlighting the individualized prescription. Discussion: The positive effects of prescribed physical exercise are confirmed, on the symptoms of depression and anxiety of various populations, being effective and inexpensive, which opens the need to prioritize it and apply its principles to activities such as taichi and/or yoga to improve their effectiveness.
\end{abstract}

Keywords: Anxiety; depression; exercise; physical therapy specialty; mental health; conduct disorder

\section{Introducción}

La salud mental a nivel mundial tiene una alta incidencia de morbilidad y mortalidad por la alteración de trastornos de ansiedad y depresión, las cuales se encuentran ubicadas entre las diez primeras problemáticas de salud a nivel mundial (Organización Mundial de la Salud [OMS], 2013). Se estima que para el 2020 la prevalencia de las enfermedades cardiovasculares será superada por las enfermedades mentales asociadas a depresión y ansiedad (Murray, 1997). Los trastornos de ansiedad y depresión son los problemas más comunes en la salud mental y son producto de eventos traumáticos o enfermedades que condicionan la vida de las personas y que transforman su imagen corporal (Vaquero et al., 2013). Además afectan a más de trescientos millones de personas a nivel mundial, sin distinguir el ciclo vital, condición social o situación económica del país, provocando angustia mental y afectando la capacidad de las personas para llevar a cabo tareas cotidianas simples, generando efectos nefastos sobre las relaciones sociales (OMS, 2017).

Tal como se evidencia en la Encuesta Nacional de Salud Mental, realizada por el Ministerio de Salud de Colombia en el 2015, en promedio 10 de cada 100 adultos de 18 a 44 años tienen una enfermedad mental y que 12 de cada 100 adolescentes evidencian signos y síntomas de ansiedad y depresión, relacionados a consumo de alcohol, sustancias psicoactivas y experiencias traumáticas (Ministerio de Salud y Protección Social, 2017; ICBF, 2014).

Este tipo de trastornos inciden principalmente a niños, adolescentes y adultos jóvenes afectando la productividad en sus diferentes contextos, factores personales y sociales. Los primeros trastornos depresivos se presentan a los 24 años y el de ansiedad entre los 7 y 28 años con una edad promedio de 17 años, relacionado en un 73\% al uso de sustancias psicoactivas o rechazo social (Posada, 2013). 
De acuerdo al estudio realizado por Harvath y McKenzie (2012), la sociedad actual está expuesta a factores intrínsecos y extrínsecos desencadenantes de ansiedad y depresión; siendo esto un síntoma de una condición de la persona o una enfermedad en sí misma. No obstante, la incidencia y prevalencia de estas condiciones son subvaloradas, subdiagnosticadas y por ende subtratadas, aumentando los efectos sobre la calidad de vida de la persona y por ende sobre la sociedad.

La ocurrencia de estas enfermedades (diagnosticadas o no) aumenta considerablemente en personas con antecedentes de eventos traumáticos asociados a factores psicosociales, cuadros de estrés, cambios hormonales, enfermedades, periodo gestacional y abuso de sustancias psicoactivas; causando una serie de cambios biológicos y psicológicos relacionados ya sea con el ambiente o el entorno en el que interactúa la persona (Firts, Frances, \& Picus, 2003).

A pesar de los beneficios del ejercicio en la salud mental y el control de los trastornos psíquicos y su correlación con inhibición de la estimulación del sistema nervioso simpático y enfermedades asociadas a la depresión, ansiedad y al estrés (Musil et al., 2018), es una práctica que no se aplica comúnmente como estrategia preventiva o de tratamiento médico o terapéutico; puesto que la administración de medicamentos sigue siendo el primer recurso para tratar dichos trastornos (Garcia \& Martin, 2014).

Por lo cual, esta investigación buscó determinar los efectos y beneficios del ejercicio físico sobre los trastornos de la salud mental en las variables de depresión y ansiedad en seres humanos, bajo la revisión de evidencia científica de calidad, con la finalidad de: 1 . Obtener evidencia científica de los beneficios propios de la práctica de ejercicio físico en personas que presentan problemas de salud mental relacionados con la depresión y la ansiedad, 2. Establecer los efectos del ejercicio físico, en el estado físico, emocional y psicosocial, de las personas con problemas de salud mental teniendo en cuenta la variable de tiempo, 3. Identificar los efectos del ejercicio físico teniendo en cuenta el tipo de intervención, de manera estructurada en personas con diagnóstico de depresión y ansiedad.

\section{Método}

Se llevó un acabo una revisión sistemática de literatura científica de calidad, determinada por ensayos clínicos que permitan identificar los beneficios del ejercicio físico sobre la salud mental, en las variables de ansiedad y depresión en personas mayores de 16 años.

Se realizó una búsqueda a nivel mundial en las siguientes bases de datos Redalyc, PubMed, EbscoHost, Elsevier, PeDro, DialNet, Scielo y ATGC (pubmed) de ensayos clínicos aleatorizados y controlados, tomando las publicaciones desde el año 2000 en adelante, ya que a partir de este año se encontraron artículos que cumplían con los parámetros de calidad, que involucran el ejercicio y la salud mental en población joven no menor de 16 años en adelante. Los artículos fueron seleccionados en idiomas inglés, ruso, francés, español, alemán y portugués, utilizando los siguientes términos MeSh: Anxiety, Depression Physical exercise, Cancer.

Se tomaron en cuenta todos los artículos científicos publicados, que vinculen dentro de su población de estudio a personas con trastornos depresivos o ansiosos los cuales se encuentren respaldados por un diagnóstico médico. Se tomó en cuenta Ensayos Clínicos que involucraran personas mayores de 16 años, sin discriminación de edad, género, sexo o nivel socioeconómico. Se excluyó todo el material académico que no fuera publicado en revistas científicas, se descartaron artículos cuya metodología no permiten identificar los protocolos. 
Para la identificación, extracción y descripción de los estudios, se requirió del trabajo en equipo de 3 evaluadores externos, los cuales utilizaron 3 matrices de búsqueda en donde se identifican las características de los artículos para facilitar la evaluación de los mismos. Matriz 1: con los criterios aceptado, rechazado y pendiente; para este último, se tomó la decisión de llevar al 3 evaluador. Matriz 2: vinculó la información de autor, título, año de publicación, doi, aleatorización, ocultamiento, cegamiento, tamaño de la muestra, número de brazos, tipo de estudio, tipo de intervención, métodos de evaluación y resultados. Matriz 3: especificó las variables del artículo para determinar el grado de calidad del mismo, incluyendo los parámetros de prescripción.

\section{Resultados}

La búsqueda arrojó un total de 73574 artículos que al discriminar por variables de la siguiente manera y base de datos resulta un número variable de artículos tal y como se observa en la figura 1.

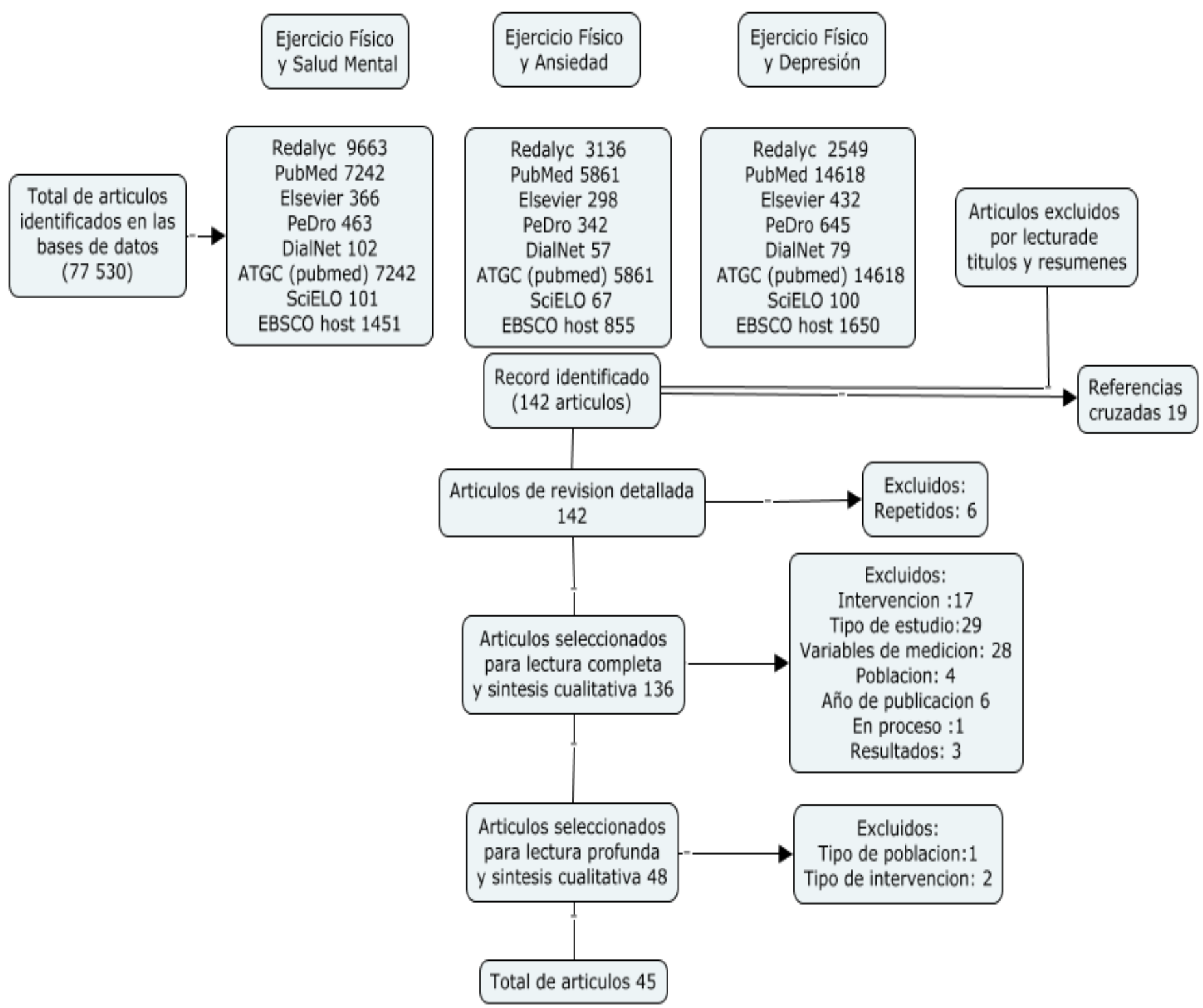

Figura 1. Diagrama de flujo de la selección de estudios. 
Un número de estudios (44) cumplieron con los criterios de inclusión, los cuales fueron publicados en los siguientes países: 10 de Estados Unidos, 6 de Canadá, 4 de Brasil, 4 de Alemania, 3 de Dinamarca, 3 de Irán, 3 de Taiwán, 2 de Australia y 1 de Nigeria, Sudáfrica, Suecia, Italia, Reino Unido, Bélgica, Portugal, Noruega y España, respectivamente (ver Tabla 1).

Tabla 1.

Artículos incluidos en el estudio.

\begin{tabular}{|c|c|c|c|}
\hline Autor & País & Año & Población \\
\hline (Andersen et al., 2013) & Dinamarca & 2013 & 91 \\
\hline (Quist et al., 2015) & Dinamarca & 2015 & 114 pacientes (57 mujeres, 57 hombres) \\
\hline $\begin{array}{l}\text { (Aweto, Aiyegbusi, Ugonabo, \& } \\
\text { Adeyemo, 2016) }\end{array}$ & Nigeria & 2016 & 40 \\
\hline (Balchin et al., 2016) & Sudáfrica & 2016 & 30 \\
\hline $\begin{array}{l}\text { (Jaworska, Courtright, De Somma, } \\
\text { MacQueen, \& MacMaster, 2018) }\end{array}$ & Canadá & 2018 & $13(18+)$ \\
\hline (Paolucci et al., 2018) & Canadá & 2018 & 61 adultos \\
\hline (Danielsson et al., 2014) & Suecia. & 2014 & 62 adultos con medicación antidepresiva \\
\hline (Murri et al., 2018) & Italia & 2018 & 121 \\
\hline (Nia et al., 2011) & Irán & 2011 & 40 \\
\hline (Lamego et al., 2016) & Brasil & 2016 & 10 \\
\hline (J.-E. Yang et al., 2017) & Taiwán & 2017 & 140 mujeres postnatales \\
\hline (Smith et al., 2010) & Hawái & 2010 & 30 \\
\hline (Oertel-Knöchel et al., 2014) & Germania & 2014 & 51(28 mujeres-23 hombres) \\
\hline (Knubben et al., 2006) & Germania & 2006 & 38 \\
\hline (Atlantis et al., 2004) & Australia & 2004 & $\begin{array}{l}73 \text { participantes (mujeres }=37 \text {, hombres } \\
=36 \text { ) }\end{array}$ \\
\hline (Haglund et al., 2014) & EEUU & 2014 & Los participantes fueron 135 adultos \\
\hline (LeBouthillier \& Asmundson, 2017) & Canadá & 2017 & $\begin{array}{l}\text { Un total de } 48 \text { individuos entre } 18 \text { y } 65 \\
\text { años }\end{array}$ \\
\hline $\begin{array}{l}\text { (Dehghan-nayeri \& Adib- } \\
\text { Hajbaghery, 2011) }\end{array}$ & Canadá & 2011 & 200 Estudiantes \\
\hline (StröHle et al., 2007) & Germania & 2007 & 2548 \\
\hline $\begin{array}{l}\text { (Pourmousavi Khoshnab \& } \\
\text { Nikseresht, 2016) }\end{array}$ & Irán & 2016 & 60 Mujeres \\
\hline (Midtgaard et al., 2005) & Dinamarca & 2005 & 91 \\
\hline (Wang et al., 2018) & EEUU & 2018 & 226 \\
\hline (Chen, Tsai, Wu, Lin, \& Lin, 2015) & Taiwan & 2015 & 116 \\
\hline (Daley et al., 2015) & Reino Unido & 2015 & 146 \\
\hline (Rawson et al., 2015) & EEUU & 2015 & 135 \\
\hline (Suh et al., 2002) & Taiwan & 2015 & 14 \\
\hline (Vancampfort et al., 2011) & Belgica & 2011 & 64 \\
\hline (Felipe José Aidar et al., 2012) & Portugal & 2012 & 24 \\
\hline (Felipe J. Aidar et al., 2013) & Brasil & 2013 & 31 \\
\hline (Eriksen et al., 2002) & Noruega & 2002 & 860 personas \\
\hline (Penninx et al., 2002) & EEUU & 2002 & 439 personas \\
\hline (Annesi \& Vaughn, 2011) & EEUU & 2011 & 88 Personas \\
\hline (Courneya et al., 2014) & Canadá & 2014 & 301 pacientes \\
\hline (Fetzner \& Asmundson, 2015) & Canadá & 2015 & 33 pacientes \\
\hline (Jazaieri et al., 2012) & EEUU & 2012 & 56 personas \\
\hline (Smits et al., 2008) & EEUU & 2008 & 60 personas \\
\hline
\end{tabular}




\begin{tabular}{lcll}
\hline (El-Shamy, 2013) & EEUU & 2012 & 30 personas \\
\hline (Chan, Immink, \& Hillier, 2012) & Australia & 2012 & 30 personas \\
\hline (Aidar et al., 2018) & Brasil & 2017 & 36 personas \\
\hline (Pomp et al., 2013) & Alemania & 2013 & 361 \\
\hline (Bonet et al., 2017) & España & 2017 & 30 estudiantes universitarios \\
\hline (Branco et al., 2015) & Brasil & 2015 & 26 personas \\
\hline (Marco et al., 2010) & Irán & 2010 & 86 mujeres \\
\hline (Cox et al., 2004) & EEUU & 2003 & 24 mujeres \\
\hline
\end{tabular}

Dada las diversas intervenciones con efectos en la ansiedad y depresión, se realizó una división por subgrupo debido a la diversidad de factores etiológicos y nivel de depresión y ansiedad, divididos de la siguiente manera: estrés postraumático, origen ocupacional, drogodependencia, trastornos mentales, enfermedades diagnosticadas (patologías), conductas según la edad y ginecobstetricias.

\section{Análisis por Subgrupos}

Drogodependencia. En el trabajo de Haglund et al. (2014) realizaron intervenciones de ejercicio con una duración de 60 minutos por 3 días a la semana en donde se trabajó la fuerza, capacidad aeróbica y flexibilidad, y encontraron que hubo mejoras significativas en la reducción de la depresión medida con BDI, en una población drogodependiente en proceso de rehabilitación, adicional a esto expone los resultados tuvieron menor significancia en aquellos usuarios que consumen Metanfetamina.

Rawson et al. (2015) plantearon un estudio que evalúa el impacto del ejercicio físico en los síntomas sobre la depresión y la ansiedad en personas dependientes de metanfetaminas, se realizó en una población de 135 personas dependientes, que fueron evaluadas con el Inventario de Depresión de Beck y el Inventario de Ansiedad de Beck para medir los síntomas. La realización de ejercicios tuvo una duración de 8 semanas que constaba de ejercicio aeróbico 3 veces por semana y 60 minutos durante 8 semanas con una duración de 55 min: calentamiento de $5 \mathrm{~min}, 30 \mathrm{~min}$ de actividad aeróbica en una cinta de correr, 15 min de entrenamiento con pesas, 5 min de estiramiento entre el 60-80\% de FC; por otro lado, contaba con un grupo control al cual se le realizó sesiones estructuradas de educación para la salud 3 días a la semana durante 8 semanas con una duración de 55 minutos se encontró una disminución en los participantes que realizaron el programa de 8 semanas de ejercicio físico de los niveles de depresión $(\beta=-0.63, \mathrm{P}=0.001)$ y ansiedad $(\beta=-0.95, \mathrm{P}=0.001)$ puntajes finales, $\mathrm{y}$ una disminución significativa entre la dosis y los síntomas de depresión $\beta=-0.61, \mathrm{P}<0.001)$ ansiedad $(\beta=-0.22, \mathrm{P}=0.009)$ lo que concluye que un programa de ejercicios estructurado permite disminuir los síntomas de depresión y ansiedad en personas dependientes de metanfetamina.

Trastornos Mentales. Este tipo de trastornos afectan cada vez más a la población debido a múltiples factores como la edad, eventos, situaciones traumáticos que ocasionan un cambio emocional en la persona y su contexto, es así como se plantean diferentes estudios para el manejo de los síntomas que ocasionan estos trastornos, no solo de manera farmacológica, si con el ejercicio físico. Atlantis, Chow, Kirby y Singh (2004), realizaron una intervención con ejercicio físico durante 24 semanas de duración, en donde se logró obtener mejoras en las variables de estado psicológico y calidad de vida; evaluadas con la encuesta de estado de salud SF36, depresión y ansiedad evaluadas con el cuestionario DASS, y el cuestionario de BECK. Penninx et al.,(2002) trabajaron con 3 grupos, el primero con ejercicio aeróbico durante 60 minutos, con una frecuencia de una vez por semana en un periodo de 15 meses, cuya estructura disponía de 10 minutos de calentamiento, 40 minutos de marcha a una intensidad del 50\%-70\% de la Fc Max y 10 minutos de trabajo de flexibilidad; en el segundo grupo, utilizó protocolo 
de fuerza resistencia con una frecuencia de 3 veces a la semana durante 60 minutos, donde 10 minutos eran calentamiento, 40 se dedican a trabajo de fuerza resistencia utilizando como medio pesas para realizar series de 10 a 12 repeticiones y 10 minutos para el trabajo de flexibilidad, en relación al grupo control, en el cual no se realizó ningún tipo de intervención, se observó que la depresión medida con la escala CES-D11 aumentaba en promedio un 2\%, mientras que en el grupo de ejercicio aeróbico se disminuyó el puntaje en un $23 \%$ siendo disminuciones significativas de $\mathrm{P}=0,001 \%$, en cambio en el grupo intervenido con fuerza resistencia se logró una reducción en el puntaje de la escala de $6 \%$ pero esta no fue significativa.

Jazaieri, Goldin, Werner, Ziv, y Gross, (2012), encontraron que los niveles de ansiedad social medidos con la escala LSAS-SR se han reducido de manera significativa al igual que la ansiedad a la interacción medida con la escala SIAS-S con una intervención de 3 sesiones de ejercicio físico semanales a una intensidad moderada durante 8 semanas, adicionalmente también demostraron una disminución significativa en el puntaje de la escala BDI con la cual se mide la depresión. Por otro lado, Smits et al. (2008) encontraron una disminución significativa en los niveles de ansiedad medidos con el inventario de ansiedad de Beck y el índice de Spielberg, al igual que en el índice de depresión de Beck demostrando que las personas eran menos sensibles a los síntomas de estos trastornos al practicar ejercicio físico, el protocolo de intervención realizado era de 6 sesiones de ejercicio físico de 20 minutos de duración con una frecuencia de 3 veces por semana.

La investigación realizada por El-Shamy (2013), expone que los participantes tuvieron una mejora en la calidad del sueño, evaluado con la escala MDSS, así mismo también disminuyó los índices de depresión evaluados con la escala de SDS la cual parte de la autopercepción del nivel de depresión del participante, realizando un protocolo de intervención de 2 meses de duración donde se realizó ejercicio físico de tipo aeróbico 2 veces por semana, cuya estructura incluye calentamiento, trabajo de resistencia aeróbica con una intensidad del 60\%-70\% medida con la Fc Máx. en 50 minutos y trabajo de flexibilidad durante 5 minutos.

Nia et al., (2011) evaluaron el efecto de un programa de 8 semanas para estudiantes sobre la imagen corporal y la ansiedad, para ello se realizó entrenamiento en fuerza de circuito 3 días por semana, a parte un grupo control de sujetos que no participaron en ninguna actividad de entrenamiento para la medición de ansiedad se utilizó el Inventario de ansiedad del rasgo estado de Spielberg, obteniendo como resultado una reducción significativa en ansiedad $(36.40 \pm 10.32)$ en la semana 8 (31.55 \pm 8.21$)$ $(\mathrm{p}<0.05)$ y un aumento de la imagen corporal $(\mathrm{p}<0.05)$ demostrando que un programa de 8 semanas mejora significativa la imagen corporal y se obtienen reducciones significantes en los síntomas de ansiedad.

Knubben et al., (2006) realizaron un estudio que evaluó los efectos a corto plazo del ejercicio en pacientes con depresión mayor en una población de 39 pacientes mediante una intervención de ejercicios de resistencia: caminata diaria en una cinta de correr durante 10 días. Se aplicó las escalas de melancolía Besh-Rafaelsen (BRMS) y la escala de depresión del Centro de Estudios Epidemiológicos (CES-D) los resultados obtenidos fueron la reducción de puntajes en depresión después de 10 días en el grupo de ejercicio BRMS: $36 \%$ 18\%; CES-D: $41 \%$ v 21\%; $\mathrm{p}=0,01$, esto demuestra que un programa a corto plazo ayuda a mejorar los estados de ánimo en pacientes con depresión mayor.

Enfermedades diagnosticadas (Patologías). Diversas patologías generan una disminución del componente físico, psicológico y social, asociado a las consecuencias de las mismas y sus tratamientos, generando una alteración de la salud mental en relación con la ansiedad y depresión, es por esta razón 
que diversos autores validan el ejercicio como una estrategia de intervención no invasiva. Andersen et al. (2013) realizaron un estudio en el que evaluaron los efectos del ejercicio físico y la fatiga durante 6 semanas en pacientes sometidos a quimioterapia, en 9 horas por semana y 4 días. En cada sesión se realizó 30 minutos de calentamiento y se finalizó con estiramientos y ejercicios de coordinación, encontrando mejoras significativas en la reducción de los niveles de fatiga $(\mathrm{p}=0,002)$ evaluados con el cuestionario (FACT - An) encontrando una correlación con mejoras en la depresión $\mathrm{p}=0.046$, pero no con las mejoras en la ansiedad, $\mathrm{p}=0.462$.

Por otro lado, Quist et al. (2015) llevaron a cabo un estudio en pacientes con cáncer de pulmón en un estadio avanzado, realizando una intervención de ejercicio físico y de relajación durante 1 hora y 50 minutos 2 veces por semana en un periodo de 6 semanas; se realizó una fase de calentamiento, fuerza, capacitación y estiramiento, demostrando una reducción en el nivel de ansiedad, evaluado con la escala HADS ( $\mathrm{p}=0.0007$ ), Así como una mejoría en el parámetro de bienestar emocional (FACT-L) y un aumento significativo de la condición física $(\mathrm{P}<.001)$. Por otro lado, el estudio realizado por Midtgaard et al. (2005) en personas bajo quimioterapia, demostró que el ejercicio multidimensional de baja y moderada intensidad, disminuye la ansiedad ( $\mathrm{p}<0.001)$ y la depresión $(\mathrm{p}=0.042)$. Así mismo, Courneya et al., (2014) realizaron un protocolo de intervención para pacientes oncológicos con ejercicio aeróbico basado en las recomendaciones de la OMS encontrando disminuciones significativas en las variables de depresión $\mathrm{P}=0.36$, Ansiedad $\mathrm{P}=0.35$ Estrés percibido $\mathrm{P}=0.41$ y Autoestima $\mathrm{P}=0.33$ todas evaluadas con la escala de síntomas depresivos CES-D11.

Wang et al., (2018) ejecutaron un estudio en pacientes con fibromialgia, realizando una intervención con ejercicio aeróbico durante 24 semanas, 2 veces por semana durante 60 minutos, iniciando con movimientos de baja intensidad y estiramientos dinámicos con intensidad entre el 60 y $70 \%$ de la FC Max. Se demostró que el ejercicio mejora en los síntomas de depresión y ansiedad, pero los grupos de Taichí combinados tuvieron mejoras estadísticamente significativas, aún más que el grupo de ejercicio aeróbico en las puntuaciones de impacto de la fibromialgia, evaluado con la escala FIQR $(\mathrm{P}=0.03)$, así como en la evaluación global del paciente $\mathrm{P}=0.005$, ansiedad evaluado con la escala HADS $(\mathrm{P}=$ 0.006), escala de autoeficacia y artritis $(P=0.0004)$ y estrategias de afrontamiento $(P=0.005)$. En relación con el tratamiento con Taichí comparado con el ejercicio aeróbico tuvo un mayor beneficio entre las puntuaciones de FIQR $(\mathrm{P}<0.001)$.

Aidar et al. (2013) evalúaron el efecto de un ejercicio acuático sobre la depresión y el estado ansiedad en sujetos que sufrieron ACV isquémico, el cual se implementó en 12 semanas, 2 veces por semana, con una duración de 45-60 min distribuida en 5-10 minutos de caminata en piscina, 5-10 min pedaleando, trabajar con spaghetti, 5-10 min escalada y descendiendo de grados de piscina 5-10 min de ejercicios para extremidades superior e inferior, con material educativo, ejercicios de respiración haciendo burbujas en el agua, 10 min de natación y 5 min de ejercicios de baja intensidad, se usó la escala de Borg entre los 12-17; en el grupo control se comenzaron las actividades 4 meses después, evaluado con las escalas de Inventario de Depresión de Beck (BDI) y Trace State Anxiety Inventory (IDATE), los resultados pre y pos tratamiento mostraron diferencias significativas para depresión y ansiedad ( $\mathrm{p}<0.05)$ una terapia acuática mejora los niveles de depresión y ansiedad en ACV.

Los estudios de Suh et al. (2002), Smith et al. (2010), Vancampfort et al. (2011), y Oertel-Knöchel et al. (2014), plantearon intervenciones multivariadas de comportamiento progresivo, basado en ejercicio aeróbico, entrenamiento de la fuerza muscular y la vinculación de actividades más dinámicas en el contexto de la persona con enfermedades crónicas, en donde mostraron diferencias significativas para depresión (44.8 +/- 8.4 a $39.7+/-6.4, \mathrm{P}=0.073)$, mejora significativa de la calidad de vida $(124.5+/-$ 
16.5 a 133.6+/-19.3, $\mathrm{P}=0.031)$, menores riesgos de síntomas depresivos $(\mathrm{OR})=0.52$, con un intervalo de confianza (IC) del 95\% $=0.32-0.83, p=0.006$ y OR $=0.61$, IC del 95\% $=0.39-0.97, p=0.04$ y una mayor relajación muscular progresiva un puntaje de 1,26 para el bienestar subjetivo y -1.25 y -1.02 para la ansiedad y el estrés psicológico respectivamente.

Eng y Reime (2014) desarrollaron una revisión sistemática y metanálisis sobre los efectos del ejercicio físico en pacientes con depresión posterior a un accidente cerebrovascular, mostrando una disminución en los síntomas después de finalizar el programa de ejercicios con un valor $\mathrm{p}>0.03$; sin embargo, esta disminución de los síntomas no se mantuvieron a largo plazo, mientras que la población que realizó ejercicio físico de manera constante a una intensidad moderada-vigorosa, tuvo un efecto positivo en la etapa de recuperación subaguda ( $\leq 6$ meses después del ictus) como crónica ( $>6$ meses), contrario a aquellos que realizaron ejercicio físico a una intensidad leve. Adicionalmente, Teruel, Ruano y Moles (2012) muestran cómo el ejercicio físico mejora la condición de vida de las personas con el trastorno mental severo, al mejorar la ejecución de las actividades de la vida diaria, la disminución de los niveles de agitación, y conductas de depresión; resaltando la importancia de los fisioterapeutas a la hora de generar estrategias para incentivar a los pacientes con enfermedades mentales, los cuales suelen tener dificultades con la concentración, baja motivación, paranoia, preocupación, producto de sus patologías o efectos secundarios de los medicamentos.

Así mismo, es importante resaltar la influencia que tiene las enfermedades al condicionar la vida de los cuidadores, su familia y/o su entorno social, ya que tal como lo mostraron Marco et al. (2010) al realizar su estudio transversal, existe una influencia directa en la salud mental y los niveles de depresión, los cuales fueron valorados con sub escalas de vitalidad $(\mathrm{p}=0,003)$, función social $(\mathrm{p}=0,01)$, rol emocional $(\mathrm{p}=0,038)$ y salud mental $(\mathrm{p}=0,002)$.

Conductas según la edad. Bonet, Parrado y Capdevila (2017) realizaron un programa de ejercicio físico con una intensidad del $80 \%$, en estudiantes universitarios demostrando que las personas que realizan ejercicio físico desde hace más de 1 mes, presentan menos síntomas de depresión $(\mathrm{p}<0.001)$, con una diferencia significativa de los síntomas depresivos, entre los Activos y No activos ( $\mathrm{p}=0.003$ ), con un aumento significativo en el post-ejercicio para el total de la muestra. (Balchin, Linde, Blackhurst, Rauch, \& Schönbächler, 2016) demostró cómo el ejercicio de baja intensidad no genera una liberación significativa de endorfinas a diferencia del ejercicio a moderada y a alta intensidad con un nivel de significancia de ( $\mathrm{p}=0.07)$, lo que se evidencia en la disminución de los síntomas de depresión, pánico y miedo evaluados con la escala DASS y Pánico con Escala Self Report (PDSS-SR)

En un estudio realizado por Paolucci, Loukov, Bowdish, y Heisz en el 2018, con estudiantes universitarios, se evidencia una relación entre el ejercicio físico y la ansiedad; encontrando que la depresión disminuyó significativamente con un valor $(\mathrm{p}=.005)$ al realiza una intervención de ejercicio físico a una intensidad moderada y un método continuo en comparación a un ejercicio de alta intensidad con un método interválico. Danielsson et al. (2014) y Murri et al. (2018) determinaron que el ejercicio aeróbico en compañía de ejercicios de conciencia corporal, de estabilización postural y respiración, modulan la intensidad y frecuencia de episodios de ansiedad $(\mathrm{p}=0.06)$ incidiendo sobre la agitación y penetración $(\mathrm{p}=0.5)$.

Cox, Thomas, Hinton, y Donahue (2004) realizaron un estudio en la población femenina, en donde realizaron ejercicios con una duración de 33 minutos, durante una sesión de entrenamiento, mediante ejercicio sub máximo, encontrando una disminución significativa de la ansiedad estado ( $p<0.0001$,) evaluada con el Inventario de Ansiedad Estado (STAI) y una interacción entre la Intensidad y la edad 
$(p=0.04)$. De igual forma, Pomp, Fleig, Schwarzer y Lippke (2013) demostraron que los ejercicios de rehabilitación durante las 6 semanas disminuyen síntomas depresivos $(\mathrm{p}=0.005)$ evaluados con la escala de Estado de ánimo (PHQ-2) en el grupo de intervención, sin encontrar diferencias significativas en el grupo control.

En el artículo de Yang, Lee y Kim, (2017) se trabajó con una muestra poblacional de adultos mayores, realizando actividad física mediante realidades virtuales, en donde se evidenció un cambio en la depresión y estrés después del ejercicio. La puntuación de GDS-K se redujo después de la intervención al igual que el puntaje ISS-K después de la intervención ( $\mathrm{p}<0.05)$. De igual forma, el estudio realizado por Branco et al. (2015) en esta misma población, promueve una mejora de la salud física y psicológica de las personas mayores por medio de ejercicio físico, encontrando una mejora significativa en las post prueba sobre los síntomas depresivos $(\mathrm{p} \leq 0.001)$ evaluados con Escala de Depresión Geriátrica (GDS), en todas las pruebas de aptitud física $(\mathrm{p} \leq 0.05)$ evaluadas con Senior Fitness Test y una reducción de la circunferencia de la cintura, teniendo efectos positivos de la intervención $(\mathrm{p}=0.031)$.

Ginecobstetricos. Los trastornos mentales que afectan antes, durante y después del parto (Castellón, 2012) generaron alteraciones del estado de ánimo, principalmente entre la cuarta hasta la semana 30 , afectando la relación madre e hijo y el desarrollo durante la gestación al igual que la calidad de vida de la madre. Muy pocos estudios evalúan trastornos en el parto, por lo cual Yang y Chen (2018) utilizaron una muestra de 140 mujeres en quienes se le evaluaron los niveles de ansiedad con la escala de depresión posnatal de Edimburgo (EPDS) La intervención consta de ejercicio gimnástico aeróbico al menos tres veces (15 minutos) por semana durante tres meses con un disco compacto en el hogar, el grupo control recibió atención postparto regular. El grupo intervención mostró resultados significativos para ansiedad EPDS en el grupo intervención 1.48 ( $<<0.05) 1.05$ ayudando a mejorar el estado de ánimo de las mujeres.

Por otro lado Daley et al. (2015) realizan un estudio en una población de mujeres (146 ) que cuenten con la Clasificación Internacional de Enfermedades (ICD) -10 para la depresión mayor en los primeros 6 meses postnatales, en donde aplicaron la Escala de Depresión Postnatal de Edimburgo (EPDS) para la medición de depresión, como intervención se realizó 1-12 semana 30 min de ejercicio con intensidad moderada en 3 días por semana durante la semana 13-21 y 30 min de ejercicio con intensidad moderada de 3-5 días por semana, incluye 2 consultas de ejercicio personalizado, durante el 1 y 2 mes recibieron llamadas telefónicas, el grupo control contó con atención habitual, consultar a su médico y recibir tratamiento activo. Se obtuvo mejoras en el grupo de intervención en cuanto a la escala de depresión (46.5\% v. $23.8 \%, \mathrm{p}=0.03)$ demostrando que el ejercicio favorece la disminución de los síntomas en la depresión postnatal, y que ayuda a mejorar el estado de ánimo de las mujeres que lo presentan.

Estrés postraumático y origen ocupacional: LeBouthillier y Asmundson (2017) reunieron una población de 48 estudiantes con diagnóstico de ansiedad encontrando mejoras significativas en las variables de ansiedad, sin embargo los estudios de Fetzner y Asmundson (2015), de Jindani et al. (2015), y de Eriksen et al. (2002) no encontraron cambios significativos para estas variables. 


\section{Discusión}

Esta investigación resaltó cuatro tendencias principales para el abordaje del ejercicio físico en depresión y ansiedad: a. prescripción del ejercicio físico de manera individualizada teniendo en cuenta la condición de salud de la persona, las necesidades, virtudes y objetivos de cada individuo, b. el uso de recursos tecnológicos a través de aplicaciones que permiten controlar la duración de las actividades y la optimización de componentes de equilibrio, coordinación y agilidad, c. ejercicio físico basado en métodos grupales y convencionales en donde se brinda una asesoría general, y d. ejercicio con técnicas complementarias como el Taichí y el Yoga , así como intervenciones acuáticas y en tierra, en las cuales se evidencian pocos cambios significativos.

La mayoría de estudios proporcionaron resultados positivos en cuanto a la disminución de los síntomas de depresión y ansiedad mediante la práctica del ejercicio. Como consecuencia se encontraron resultados efectivos que indican que el ejercicio físico mejora los trastornos mentales a los que se ven expuestos infinidad de personas; sin embargo, de estas cuatro tendencias, la prescripción individualizada presenta mejores resultados que las actividades grupales.

Lo anterior evidencia que el ejercicio, no solo logra disminuir los niveles de ansiedad y depresión a bajos costos, sino que también es una estrategia que permite brindar una mayor accesibilidad. De acuerdo con la evidencia encontrada, existen varios tipos de intervención del ejercicio físico para disminuir la ansiedad y depresión, sin embargo, de acuerdo a los resultados encontrados, la especificidad es fundamental para aumentar el impacto sobre la ansiedad y depresión. Mostrando la importancia del ejercicio físicos como terapia complementaria a la farmacológica (Villamil, 2018). Es necesario seguir profundizando en los beneficios que el ejercicio físico proporciona, facilitando así la práctica del ejercicio de una manera estructurada, siendo una estrategia para reducir los síntomas de depresión y ansiedad, mejorar la calidad de vida y contribuir a que más individuos accedan a la práctica del ejercicio y no solo al uso de fármacos o métodos invasivos para tratar los signos y síntomas de las alteraciones de la salud mental.

Los resultados demuestran que el ejercicio físico, no solo beneficia a las personas que lo practican, sino que también contribuye a mejorar el entorno en el que se desenvuelven, contribuyendo al óptimo desempeño de sus roles sociales. Así mismo, se promueven cambios psicológicos, que favorecen su estado de ánimo de su contexto el cual se ve alterado por la modificación de su dinámica natural (Marco et al., 2010) previniendo y controlando la aparición de enfermedades crónicas no transmisibles (ECNT), y ayudando al sistema músculo esquelético al depositar mayor proporción de minerales que favorecen el soporte de la carga del mismo. (Arrébola, 2001). Adicionalmente el ejercicio fomenta el fortalecimiento del sistema cardiovascular mejorando la capacidad de transporte de oxígeno.

El siguiente desafío es establecer el ejercicio como herramienta terapéutica transversal a la condición de salud (Villamil \& Hernandez, 2018; Villamil, 2018); por lo cual es necesario investigar qué tan alto es el índice de adherencia a hábitos en las personas que sufren estos síndromes y las estrategias para mejorar los estilos de vida de acuerdo a la población teniendo en cuenta los factores extrínseco e intrínsecos que influyen en estos, contribuyendo en la toma de decisiones ante intervenciones clínicas o incluso de Rehabilitación Basada en Comunidad (RBC), permitiendo mejorar la producción científica de calidad (Vernaza \& Alvarez, 2011) y la alta prevalencia de los síntomas, en Colombia, la cual es amplia en comparación con las estadísticas de otros países desarrollados y en desarrollo (Posada, 2013). 
Tal como muestra el Ministerio de Salud y Protección Social (2017), las atenciones por depresión se han incrementado desde el año 2009, por lo que es necesario mejorar las estrategias para tratar los síntomas, permitiendo disminuir la prevalencia e incidencia en la población colombiana, la cual presenta síntomas desde los 15 a los 64 años, con mayor incidencia en las en las edades de 45 a 59 años y una prevalencia en las mujeres, asociado a ECNT como la trombosis cerebral $(58,1 \%)$, seguida de problema psiquiátrico $(54,3 \%)$, hipertensión $(50,9 \%)$, infarto del miocardio $(50,6 \%)$ y diabetes $(50,5 \%)$, en las principales ciudades del país.

La bibliografía demuestra la efectividad del ejercicio físico en el control de los síntomas de los trastornos de ansiedad y depresión, teniendo mayores efectos en las intervenciones individualizadas, con un volumen de carga de mínimo 30 minutos (Branco et al., 2015), una duración superior a las 6 semanas (Rawson et al., 2015), intensidad entre el 40 y el $60 \%$ de la FC Max en ejercicios de capacidad aeróbica y de fuerza resistencia manteniendo una intensidad entre moderada y vigorosa (Aweto et al., 2016; Suh et al., 2002), para mantener cambios significativos en los síntomas de ansiedad y depresión. Razón por la cual es importante incentivar el desarrollo de investigaciones desde un enfoque fisioterapéutico que plantee este tipo de intervención como prioridad en la prevención y manejo de estos trastornos teniendo en cuenta sus efectos dentro de la percepción de cuerpo y la restricción que puede causar durante el desempeño de roles y actividades de la vida diaria.

En la búsqueda de artículos se encontraron algunas limitaciones, ya que muchas de las investigaciones realizaban un análisis subjetivo, no presentaban los protocolos de manera clara para lograr cambios significativos, limitando los resultados sobre las variables de depresión y ansiedad, así como la confiabilidad para replicarlos. También se evidenciaron limitaciones para acceder a la información con evidencia, ya que las bases de datos cobran por acceder a la misma, restringiendo el desarrollo a la ciencia e investigación.

\section{Agradecimientos}

Los autores agradecen a la Escuela Colombiana de Rehabilitación por su aporte a la formación y desarrollo profesional del fisioterapeuta.

\section{Conflicto de Intereses}

Los autores declaran no presentar ninguna relación de interés comercial o personal dentro del marco de la investigación que condujo a la producción del manuscrito.

\section{Colaboraciones}

Delgado Salazar Cristhian Felipe: Aporte conceptual y escrito del manuscrito, búsqueda, recolección y análisis de datos de la investigación.

Mateus Aguliera Erika Tatiana: Aporte conceptual y escrito del manuscrito, búsqueda, recolección y análisis de datos de la investigación.

Rincón Bolivar Laura Alejandra: Aporte conceptual y escrito del manuscrito, búsqueda, recolección y análisis de datos de la investigación.

Villamil Parra Wilder Andrés: Gestor intelectual de idea de investigación, director de proyecto de investigación, aporte conceptual y escrito del manuscrito, análisis de datos finales, editor del documento. 


\section{Referencias}

Aidar, F. J., de Oliveira, R. J., Silva, A. J., de Matos, D. G., Mazini Filho, M. L., Hickner, R. C., \& Machado Reis, V. (2012). The Influence of Resistance Exercise Training on the Levels of Anxiety in Ischemic Stroke. Stroke Research and Treatment 1-6. Doi: 10.1155/2012/298375.

Aidar, F. J., Garrido, N. D., Silva, A. J., Reis, V. M., Marinho, D. A., \& de Oliveira, R. J. (2013). Effects of aquatic exercise on depression and anxiety in ischemic stroke subjects. Health, 5(2), 222-228. Doi: 10.4236/health.2013.52030

Aidar, F. J., Oliveira, R. J. De, Matos, D. G. De, Chilibeck, P. D., Souza, R. F. de, Carneiro, A. L., \& Reis, V. M. (2018). A randomized trial of the effects of an aquatic exercise program on depression, anxiety levels, and functional capacity in of people who suffered an ischemic stroke. The Journal of Sports Medicine and Physical Fitness, 58(7-8),1171-1177 Doi: 10.23736/S00224707.17.07284-X

Andersen, C., Rørth, M., Ejlertsen, B., Stage, M., Møller, T., Midtgaard, J., ... Adamsen, L. (2013). The effects of a six-week supervised multimodal exercise intervention during chemotherapy on cancer-related fatigue. European Journal of Oncology Nursing, 17(3), 331-339. Doi: 10.1016/j.ejon.2012.09.003

Annesi, J. J., \& Vaughn, L. L. (2011). Relationship of Exercise Volume with Change in Depression and Its Association with Self-Efficacy to Control Emotional Eating in Severely Obese Women. Advances in Preventive Medicine, 2011, 1-6. Doi: 10.4061/2011/514271

Arrébola, P. (2001). Ejercicio y hueso: Revisión bibliográfica. Revista Española de Enfermedades Metabólicas Óseas, 10(4), 119-131

Atlantis, E., Chow, C.-M., Kirby, A., \& Singh, M. F. (2004). An effective exercise-based intervention for improving mental health and quality of life measures: a randomized controlled trial. Preventive Medicine, 39(2), 424-434. Doi: 10.1016/j.ypmed.2004.02.007

Aweto, H. A., Aiyegbusi, A. I., Ugonabo, A. J., \& Adeyemo, T. A. (2016). Effects of Aerobic Exercise on the Pulmonary Functions, Respiratory Symptoms and Psychological Status of People Living With HIV. Journal of Research in Health Sciences, 16(1), 17-21. Recuperado de https://www.ncbi.nlm.nih.gov/pubmed/27061991

Baile, J. I. (2013). ¿Qué es la imagen corporal? Cuadernos del Marqués de San Adrián, 2, 53-70. Recuperado de https://dialnet.unirioja.es/servlet/articulo?codigo=2010565

Balchin, R., Linde, J., Blackhurst, D., Rauch, H. L., \& Schönbächler, G. (2016). Sweating away depression? The impact of intensive exercise on depression. Journal of Affective Disorders, 200, 218-221. Doi:10.1016/j.jad.2016.04.030

Bonet, J., Parrado, E., \& Capdevila, L. (2017). Efectos agudos del ejercicio físico sobre el estado de ánimo y la HRV. Revista Internacional de Medicina y Ciencias de la Actividad Física y del Deporte, 17(65). 85-100. Doi:10.15366/rimcafd2017.65.006

Branco, J. C., Jansen, K., Sobrinho, J. T., Carrapatoso, S., Spessato, B., Carvalho, J., ... Silva, R. A. da. (2015). Physical benefits and reduction of depressive symptoms among the elderly: Results from 
the Portuguese «National Walking Program». Ciência \& Saúde Coletiva, 20(3), 789-795. Doi: 10.1590/1413-81232015203.09882014

Castellón, A. D. (2012). Actualizaciones en depresión posparto. Revista Cubana de Obstetricia y Ginecología, 38(4), 576-586. Recuperado de http://scielo.sld.cu/scielo.php?script=sci_arttext\&pid=S0138-600X2012000400016

Chan, W., Immink, M. A., \& Hillier, S. (2012). Yoga and exercise for symptoms of depression and anxiety in people with poststroke disability: a randomized, controlled pilot trial. Alternative Therapies in Health and Medicine, 18(3), 34-43. Recuperado de https://www.ncbi.nlm.nih.gov/pubmed/22875560

Chen, H.-M., Tsai, C.-M., Wu, Y.-C., Lin, K.-C., \& Lin, C.-C. (2015). Randomized controlled trial on the effectiveness of home-based walking exercise on anxiety, depression and cancer-related symptoms in patients with lung cancer. British Journal of Cancer, 112(3), 438-445. Doi: 10.1038/bjc.2014.612

Courneya, K. S., McKenzie, D. C., Gelmon, K., Mackey, J. R., Reid, R. D., Yasui, Y., ... Segal, R. J. (2014). A Multicenter Randomized Trial of the Effects of Exercise Dose and Type on Psychosocial Distress in Breast Cancer Patients Undergoing Chemotherapy. Cancer Epidemiology Biomarkers \& Prevention, 23(5), 857-864. Doi: 10.1158/1055-9965.EPI-13-1163

Cox, R. H., Thomas, T. R., Hinton, P. S., \& Donahue, O. M. (2004). Effects of Acute 60 and 80\% VO 2 max Bouts of Aerobic Exercise on State Anxiety of Women of Different Age Groups across Time. Research Quarterly for Exercise and Sport, 75(2), 165-175. Doi: 10.1080/02701367.2004.10609148

Daley, A. J., Blamey, R. V., Jolly, K., Roalfe, A. K., Turner, K. M., Coleman, S., .. MacArthur, C. (2015). A pragmatic randomized controlled trial to evaluate the effectiveness of a facilitated exercise intervention as a treatment for postnatal depression: the PAM-PeRS trial. Psychological Medicine, 45(11), 2413-2425. Doi: 10.1017/S0033291715000409

Danielsson, L., Papoulias, I., Petersson, E.-L., Carlsson, J., \& Waern, M. (2014). Exercise or basic body awareness therapy as add-on treatment for major depression: A controlled study. Journal of Affective Disorders, 168, 98-106. Doi: 10.1016/j.jad.2014.06.049

Dehghan-nayeri, N., \& Adib-Hajbaghery, M. (2011). Effects of progressive relaxation on anxiety and quality of life in female students: A non-randomized controlled trial. Complementary Therapies in Medicine, 19(4), 194-200. Doi: 10.1016/j.ctim.2011.06.002

El-Shamy, F. (2013). Effect of Aerobic Exercise on Postmenopausal Women with Major Depression. British Journal of Applied Science \& Technology, 3(2), 214-219. Doi: 10.9734/BJAST/2014/2667

Eng, J. J., \& Reime, B. (2014). Exercise for depressive symptoms in stroke patients: a systematic review and meta-analysis. Clinical Rehabilitation, 28(8), 731-739. Doi: 10.1177/0269215514523631

Eriksen, H. R., Ihlebaek, C., Mikkelsen, A., Grønningsaeter, H., Sandal, G. M., \& Ursin, H. (2002). Improving subjective health at the worksite: a randomized controlled trial of stress management training, physical exercise and an integrated health programme. Occupational Medicine (Oxford, England), 52(7), 383-391. Doi: 10.1093/occmed/52.7.383

Fetzner, M. G., \& Asmundson, G. J. G. (2015). Aerobic Exercise Reduces Symptoms of Posttraumatic Stress Disorder: A Randomized Controlled Trial. Cognitive Behaviour Therapy, 44(4), 301-313. Doi: 10.1080/16506073.2014.916745

Firts, M. B., Frances, A., \& Picus, H. A. (2003). Manual diagnóstico y estadístico de los trastornos mentales.

Garcia, M., \& Martin, M. (2014). Adolescencia y Sexualidad: Resolviendo el Conflicto Natura-Cultura, [Trabajo fin de grado] Facultad de Educación de Palencia Universidad de Valladolid. Recuperado 
de https://uvadoc.uva.es/bitstream/handle/10324/6759/TFGL650.pdf;jsessionid=87DBAEA76F630946C18A85EE8A8F4E3A? sequence=1

Haglund, M., Ang, A., Mooney, L., Gonzales, R., Chudzynski, J., Cooper, C. B., ... Rawson, R. A. (2014). Predictors of depression outcomes among abstinent methamphetamine-dependent individuals exposed to an exercise intervention: Predictors of Depression Response to Exercise Treatment. The American Journal on Addictions, 24(3), 246-251. Doi: 10.1111/j.15210391.2014.12175.x

Harvath, T., A., \& McKenzie, G. (2012). Depression in older adults. En: M. Boltz, E. Capezuti, T. Fulmer, D. Zwicker (Eds). Evidence-based geriatric nursing protocols for best practice. New York, USA: Springer Publishing Company

Instituto Colombiano de Bienestar Familiar [ICBF]. (2014). Impacto del conflicto armado en el estado Psicosocial de niños, niñas y adolescentes. Recuperado de https://repository.oim.org.co/handle/20.500.11788/541

Jaworska, N., Courtright, A. K., De Somma, E., MacQueen, G. M., \& MacMaster, F. P. (2018). Aerobic exercise in depressed youth: A feasibility and clinical outcomes pilot. Early Intervention in Psychiatry, 13(1):128-132. doi: 10.1111/eip.12537. Doi: 10.1111/eip.12537

Jazaieri, H., Goldin, P. R., Werner, K., Ziv, M., \& Gross, J. J. (2012). A Randomized Trial of MBSR Versus Aerobic Exercise for Social Anxiety Disorder: MBSR V. AE in SAD. Journal of Clinical Psychology, 68(7), 715-731. Doi: 10.1002/jclp.21863

Jindani, F., Turner, N., \& Khalsa, S. B. S. (2015). A Yoga Intervention for Posttraumatic Stress: A Preliminary Randomized Control Trial. Evidence-based complementary and alternative medicine : eCAM. 351746. Doi: 10.1155/2015/351746.

Knubben, K., Reischies, F. M., Adli, M., Schlattmann, P., Bauer, M., Dimeo, F., \& Ansley, L. (2006). A randomised, controlled study on the effects of a short-term endurance training programme in patients with major depression * Commentary. British Journal of Sports Medicine, 41(1), 29-33. Doi: 10.1136/bjsm.2006.030130

Lamego, M. K., Lattari, E., Sá Filho, A. S. de, Paes, F., Mascarenhas Jr., J., Maranhão Neto, G., ... Machado, S. (2016). Aerobic exercise reduces anxiety symptoms and improves fitness in patients with panic disorder. Medical Express, 3(3). Doi: 10.5935/MedicalExpress.2016.03.06

LeBouthillier, D. M., \& Asmundson, G. J. G. (2017). The efficacy of aerobic exercise and resistance training as transdiagnostic interventions for anxiety-related disorders and constructs: A randomized controlled trial. Journal of Anxiety Disorders, 52, 43-52. Doi: 10.1016/j.janxdis.2017.09.005

Macarena, L. G., \& Silvio, D. M. (2016). Imagen corporal e insatisfacción con el propio cuerpo en Trastornos de Conducta Alimentaria en adolescentes. [Trabajo Fin de Grado]. Facultad de Humanidades y Ciencias de la Educación. Universidad de Jaén. Recuperado de: http://tauja.ujaen.es/bitstream/10953.1/3528/1/Lpez_Garca_Macarena_TFG_Psicologa. pdf

Marco, E., Duarte, E., Santos, J.-F., Aguirrezabal, A., Morales, A., Belmonte, R., ... Escalada, F. (2010). Deterioro de la calidad de vida en cuidadores familiares de pacientes con discapacidad por ictus: una entidad a considerar. Revista de Calidad Asistencial, 25(6), 356-364. Doi: 10.1016/j.cali.2010.06.003

Midtgaard, J., Rørth, M., Stelter, R., Tveterås, A., Andersen, C., Quist, M., ... Adamsen, L. (2005). The impact of a multidimensional exercise program on self-reported anxiety and depression in cancer patients undergoing chemotherapy: a phase II study. Palliative \& Supportive Care, 3(3), 197208. Recuperado de: https://www.ncbi.nlm.nih.gov/pubmed/16594459 
Ministerio de Salud y Protección Social. (2017). Boletín de salud mental Depresión Subdirección de Enfermedades No Transmisibles. Recuperado de: https://www.minsalud.gov.co/sites/rid/Lists/BibliotecaDigital/RIDE/VS/PP/ENT/boletindepresion-marzo-2017.pdf

Murray CJ Lopez. (1997). Global mortality, disability, and the contribution of risk factors: Global Burden of Disease Sudy. Lancet. 349, 1463-1442. Doi: 10.1016/S0140-6736(96)07495-8

Murri, M. B., Ekkekakis, P., Menchetti, M., Neviani, F., Trevisani, F., Tedeschi, S., ... Amore, M. (2018). Physical exercise for late-life depression: Effects on symptom dimensions and time course. Journal of Affective Disorders, 230, 65-70. Doi: 10.1016/j.jad.2018.01.004

Musil, R., Seemüller, F., Meyer, S., Spellmann, I., Adli, M., Bauer, M., ... Riedel, M. (2018). Subtypes of depression and their overlap in a naturalistic inpatient sample of major depressive disorder. International Journal of Methods in Psychiatric Research, 27(1), e1569. Doi: 10.1002/mpr.1569

Nia, F. R., Arazi, H., Rahimi, R., Kurd, K. P., \& Hossaini, K. (2011). Effects of an eight-week circuit strength training program on the body images and anxiety in untrained college students. Medicina dello Sport, 63(3297), 308. Recuperado de: https://www.minervamedica.it/en/journals/medicinadello-sport/article.php?cod=R26Y2011N03A0297

Oertel-Knöchel, V., Mehler, P., Thiel, C., Steinbrecher, K., Malchow, B., Tesky, V., ... Hänsel, F. (2014). Effects of aerobic exercise on cognitive performance and individual psychopathology in depressive and schizophrenia patients. European Archives of Psychiatry and Clinical Neuroscience, 264(7), 589-604. Doi: 10.1007/s00406-014-0485-9

Organización Mundial de la Salud [OMS]. (2013). Plan de acción sobre salud mental 2013-2020. Recuperado de: https://www.who.int/mental_health/publications/action_plan/es/

Organización Mundial de la Salud [OMS]. (2017). Campañas Mundiales de la OMS: Aspectos esenciales de la campaña. Recuperado de: https://www.who.int/campaigns/world-healthday/2017/campaign-essentials/es/

Paolucci, E. M., Loukov, D., Bowdish, D. M. E., \& Heisz, J. J. (2018). Exercise reduces depression and inflammation but intensity matters. Biological Psychology, 133, 79-84. Doi: 10.1016/j.biopsycho.2018.01.015

Penninx, B. W. J. H., Rejeski, W. J., Pandya, J., Miller, M. E., Di Bari, M., Applegate, W. B., \& Pahor, M. (2002). Exercise and depressive symptoms: a comparison of aerobic and resistance exercise effects on emotional and physical function in older persons with high and low depressive symptomatology. The Journals of Gerontology. Series B, Psychological Sciences and Social Sciences, 57(2), P124-132. Doi: 10.1093/geronb/57.2.p124

Pomp, S., Fleig, L., Schwarzer, R., \& Lippke, S. (2013). Effects of a self-regulation intervention on exercise are moderated by depressive symptoms: A quasi-experimental study. International Journal of Clinical and Health Psychology, 13(1), 1-8. Doi: 10.1016/S1697-2600(13)70001-2

Posada, J. (2013). La salud mental en Colombia. Revista Biomedica, 33(4).

Pourmousavi Khoshnab, L., \& Nikseresht, A. (2016). Comparison of the Effect of Aerobic Exercise and Antidepressant Medications on Depression and Sexual Desire of Depressed Middle-Aged Women. International Journal of Women's Health and Reproduction Sciences, 5(2), 119-122. Doi: 10.15296/ijwhr.2017.22

Quist, M., Adamsen, L., Rørth, M., Laursen, J. H., Christensen, K. B., \& Langer, S. W. (2015). The Impact of a Multidimensional Exercise Intervention on Physical and Functional Capacity, Anxiety, and Depression in Patients With Advanced-Stage Lung Cancer Undergoing Chemotherapy. Integrative Cancer Therapies, 14(4), 341-349. Doi: 10.1177/1534735415572887

Rawson, R. A., Chudzynski, J., Gonzales, R., Mooney, L., Dickerson, D., Ang, A., ... Cooper, C. B. (2015). The Impact of Exercise On Depression and Anxiety Symptoms Among Abstinent 
Methamphetamine-Dependent Individuals in A Residential Treatment Setting. Journal of Substance Abuse Treatment, 57, 36-40. Doi: 10.1016/j.jsat.2015.04.007

Smith, T. L., Masaki, K. H., Fong, K., Abbott, R. D., Ross, G. W., Petrovitch, H., ... White, L. R. (2010). Effect of Walking Distance on 8-Year Incident Depressive Symptoms in Elderly Men with and without Chronic Disease: The Honolulu-Asia Aging Study: walking and 8-year incident depressive symptoms. Journal of the American Geriatrics Society, 58(8), 1447-1452. Doi: 10.1111/j.1532-5415.2010.02981.x

Smits, J. A. J., Berry, A. C., Rosenfield, D., Powers, M. B., Behar, E., \& Otto, M. W. (2008). Reducing anxiety sensitivity with exercise. Depression and Anxiety, 25(8), 689-699. Doi: 10.1002/da.20411

Suh, M. R., Jung, H. H., Kim, S. B., Park, J. S., \& Yang, W. S. (2002). Effects of regular exercise on anxiety, depression, and quality of life in maintenance hemodialysis patients. Renal Failure, 24(3), 337-345. Doi: 10.1081/jdi-120005367

Teruel, Y. C., Ruano, M. G., \& Moles, T. V. (2012). Efecto del ejercicio físico en la enfermedad mental severa [Tesis de Grado]. Facultad de Fisioterapia. Escuela Colombiana de Rehabilitación. Recuperado

de: https://repositorio.ecr.edu.co/bitstream/001/314/1/Delgado\%20Cristhian\%2C\%20Mateus\%20E rika\%2C\%20Rinc\%C3\%B3n\%20Laura.pdf

Vancampfort, D., De Hert, M., Knapen, J., Maurissen, K., Raepsaet, J., Deckx, S., ... Probst, M. (2011). Effects of progressive muscle relaxation on state anxiety and subjective well-being in people with schizophrenia: a randomized controlled trial. Clinical Rehabilitation, 25(6), 567-575. Doi: $10.1177 / 0269215510395633$

Vaquero, R., Vaquero, C., Alacid, F., Muyo, J., López, P. and Miñarro, P. (2013). Imagen corporal; revisión bibliográfica. Nutrición Hospitalaria, (1), 27-35. Doi: 10.3305/nh.2013.28.1.6016

Vernaza, P., \& Alvarez, G. (2011). Producción científica latinoamericana de fisioterapia / kinesiología. Aquichan, 11 (1), 94-107. Recuperado de: http://aquichan.unisabana.edu.co/index.php/aquichan/article/view/1820/2387

Villamil, W. (2018). Fisioterapia en cuidados intensivos, más allá del manejo respiratorio. Revista Colombiana de Rehabilitación, 17(1), 3-5. . Doi: 10.30788/RevColReh.v17.n1.2018.313

Villamil, W. (2018). Métodos de medición de la capacidad aeróbica y la fuerza muscular en pacientes con enfermedad pulmonar obstructiva crónica en un ambiente intrahospitalario. Movimiento Científico, 11(2), 55-62. Doi: 10.33881/2011-7191.mct.11202

Villamil, W., \& Hernandez Edgar. (2018). Nivel de actividad física de pacientes con falla renal pertenecientes a un Programa de Diálisis Peritoneal. Revista de Nefrología Dialisis y Trasplantel, 38(1), 28-34. Recuperado de: https://www.revistarenal.org.ar/index.php/rndt/article/view/295

Wang, C., Schmid, C. H., Fielding, R. A., Harvey, W. F., Reid, K. F., Price, L. L., .. McAlindon, T. (2018). Effect of tai chi versus aerobic exercise for fibromyalgia: comparative effectiveness randomized controlled trial. $B M J, k 851$. Doi: $10.1136 / \mathrm{bmj} . \mathrm{k} 851$

Yang, C.-L., \& Chen, C.-H. (2018). Effectiveness of aerobic gymnastic exercise on stress, fatigue, and sleep quality during postpartum: A pilot randomized controlled trial. International Journal of Nursing Studies, 77, 1-7. Doi: 10.1016/j.jinurstu.2017.09.009

Yang, J.-E., Lee, T.-Y., \& Kim, J.-K. (2017). The effect of a VR exercise program on falls and depression in the elderly with mild depression in the local community. Journal of Physical Therapy Science, 29(12), 2157-2159. Doi: 10.1589/jpts.29.2157 
Notas:

El presente artículo se derivó de la investigación titulada "Efectos del ejercicio físico sobre la salud mental (depresión y ansiedad) revisión documental”.

\section{Información de autores:}

\section{Cristhian Felipe Delgado Salazar}

Fisioterapeuta.

Escuela Colombiana de Rehabilitación

cristhiandelgado@ecr.edu.co

\section{Erika Tatiana Mateus Aguilera}

Fisioterapeuta.

Escuela Colombiana de Rehabilitación

fterikamateus@gmail.com

\section{Laura Alejandra Rincón Bolivar}

Fisioterapeuta.

Escuela Colombiana de Rehabilitación

laurarincon@ecr.edu.co

\section{Wilder Andres Villamil Parra}

Fisioterapeuta. MSc. en Fisioterapia del Deporte y la Actividad Física

Escuela Colombiana de Rehabilitación

Universidad Nacional de Colombia, sede Bogotá

wavillamilp@unal.edu.co

https://orcid.org/0000-0002-1717-1020 